\title{
Article \\ Correlation between Tissue Cellularity and Metabolism Represented by Diffusion-Weighted Imaging (DWI) and 18F-FDG PET/MRI in Head and Neck Cancer (HNC)
}

\author{
Omar Freihat ${ }^{1, *(\mathbb{D})}$, Tóth Zoltán ${ }^{2,3}$, Tamas Pinter ${ }^{4}$, András Kedves ${ }^{2,4,5}$, Dávid Sipos ${ }^{1,2,4}$, Imre Repa ${ }^{2,4}$ \\ Árpád Kovács 1,2,6,* and Cselik Zsolt ${ }^{2,7}$
}

check for updates

Citation: Freihat, O.; Zoltán, T.; Pinter, T.; Kedves, A.; Sipos, D.; Repa, I.; Kovács, Á.; Zsolt, C. Correlation between Tissue Cellularity and Metabolism Represented by Diffusion-Weighted Imaging (DWI) and 18F-FDG PET/MRI in Head and Neck Cancer (HNC). Cancers 2022, 14, 847. https://doi.org/10.3390/ cancers14030847

Academic Editors: Anna Starzyńska, Daniela Alterio and Bartosz Kamil Sobocki

Received: 17 January 2022 Accepted: 4 February 2022 Published: 8 February 2022

Publisher's Note: MDPI stays neutral with regard to jurisdictional claims in published maps and institutional affiliations.

Copyright: (C) 2022 by the authors. Licensee MDPI, Basel, Switzerland. This article is an open access article distributed under the terms and conditions of the Creative Commons Attribution (CC BY) license (https:// creativecommons.org/licenses/by/ $4.0 /)$.
1 Department of Medical Imaging, Faculty of Health Sciences, University of Pécs, 7621 Pécs, Hungary; david.sipos@etk.pte.hu

2 Doctoral School of Health Sciences, University of Pécs, 7621 Pécs, Hungary; toth.zoltan@sic.medicopus.hu (T.Z.); kedvesandras94@gmail.com (A.K.); repa.imre@sic.medicopus.hu (I.R.); cselik.zsolt@sic.medicopus.hu (C.Z.)

3 MEDICOPUS Healthcare Provider and Public Nonprofit Ltd., Somogy County Moritz Kaposi Teaching Hospital, 7400 Kaposvár, Hungary

4 Dr. József Baka Diagnostic, Radiation Oncology, Research and Teaching Center, "Moritz Kaposi” Teaching Hospital, 7400 Kaposvár, Hungary; pinter.tamas@sic.medicopus.hu

5 Institute of Information Technology and Electrical Technology, Faculty of Engineering and Information Technology, University of Pécs, 7621 Pécs, Hungary

6 Department of Oncoradiology, Faculty of Medicine, University of Debrecen, 4032 Debrecen, Hungary

7 Csolnoky Ferenc County Hospital, 8200 Veszprém, Hungary

* Correspondence: freihat.omar@etk.pte.hu (O.F.); kovacsarpad@med.unideb.hu (Á.K.); Tel.: +36-52-411-600 (Á.K.)

Simple Summary: We report on the correlation between the diffusion-weighted imaging (DWI) and the metabolic volume parameters derived from a PET scan, to determine the correlation between these parameters and the tumor cellularity in head and neck primary tumors. Our findings implied that there was no correlation between the information derived from the DWI and the information derived from the FDG metabolic parameters. Thus, both imaging techniques might play a complementary role in HNC diagnosis and assessment. This is significant because the treatment plan of patients with HNC should be well evaluated by using all the available diagnosis techniques, for a better understanding of how the tumor will react.

\begin{abstract}
Background: This study aimed to assess the association of 18F-Fluorodeoxyglucose positronemission-tomography (18F-FDG/PET) and DWI imaging parameters from a primary tumor and their correlations with clinicopathological factors. Methods: We retrospectively analyzed primary tumors in 71 patients with proven HNC. Primary tumor radiological parameters: DWI and FDG, as well as pathological characteristics were analyzed. Spearman correlation coefficient was used to assess the correlation between DWI and FDG parameters, ANOVA or Kruskal-Wallis, independent sample t-test, Mann-Whitney test, and multiple regression were performed on the clinicopathological features that may affect the 18F- FDG and apparent-diffusion coefficient (ADC) of the tumor. Results: No significant correlations were observed between DWI and any of the 18F-FDG parameters $(p>0.05)$. SUVmax correlated with N-stages $(p=0.023)$, TLG and MTV correlated with T-stages $(p=0.006$ and $p=0.001)$, and ADC correlated with tumor grades $(p=0.05)$. SUVmax was able to differentiate between $\mathrm{N}+$ and $\mathrm{N}-$ groups $(p=0.004)$. Conclusions: Our results revealed a non-significant correlation between the FDG-PET and ADC-MR parameters. FDG-PET-based glucose metabolic and DWI-MR-derived cellularity data may represent different biological aspects of HNC.
\end{abstract}

Keywords: diffusion-weighted imaging; apparent diffusion coefficient; head and neck squamous cell carcinoma; PET/MRI; glucose metabolism; primary tumor 


\section{Introduction}

Head and neck cancer is the sixth most frequent cancer worldwide, accounting for around $6 \%$ of all cancer diagnoses and approximately $1-2 \%$ of all cancer fatalities [1]. HNC cancers are a diverse collection of malignancies that are anatomically similar but differ in their origin, histology, diagnostic, and therapy techniques [2]. Squamous cell carcinomas account for $91 \%$ of all HNC cancers, sarcomas for $2 \%$, and adenocarcinomas, melanomas, and unspecified tumors for the remaining $7 \%$ [3].

Recently, ${ }^{18}$ F-fluorodeoxyglucose (FDG) positron emission tomography (PET)/magnetic resonance imaging (MRI) has emerged as an effective and accurate imaging modality in oncology [1]. PET/MRI is expected to be more valuable than PET or CT, alone or combined, because PET/MRI involves a better contrast in soft tissues and a lower radiation dose from the MRI system [1]. The advantage of clinical PET/MR is rather to replace PET/CT + MR, and reduce the radiation dose in comparison with PET/CT. DWI, a widely used technology for analyzing the motion of water molecules (Brownian motion) as a noninvasive diagnostic tool of tissue biology [4] by dissecting the texture of a biologic tissue based on the motion of water molecules at a microscopic level, is also available with PET/MRI [2]. ADC represents DWI for determining a tumor's cellularity $[3,5]$. The higher cellular tumor results in more restriction to water molecule motion which, as a result, gives lower ADC values and vice versa [4]. This means that the water molecule's motion reflects the signal loss on DWI, due to different water permeabilities through the structures [2,6]. Previous studies have proved the inversely proportional correlation between ADC and tumor cellularity [7,8]. ADC also was found to be effective in primary tumor assessment, differentiating between benign and malignant neoplasms, staging, and monitoring at post-treatment follow-up [9,10]. ADC was also found to be useful in predicting therapy response in head and neck squamous cell carcinoma (HNSCC) patients [11].

Owing to their ability to quantify glucose metabolism in tumors, FDG uptake values from PET imaging play an essential role in head and neck imaging, [12,13], which may also reflect the tumor's aggressiveness and the risk of metastasis spreading to surrounding structures $[14,15]$. SUV is the most used metric to estimate glucose metabolism, and it has shown promise for predicting the presence of metastatic lymph nodes at the original assessment, as well as survival and recurrence [16]. The metabolic parameters, TLG and MTV have emerged as new parameters that can measure the glucose metabolism activity of tumors and have been found to be more effective than SUV, because tumor contour is considered when using MTV and TLG [17]. SUVmax does not reflect the metabolic activity of the entire lesion, but measures the highest glucose metabolism in the target ROI [18]. While, MTV represents the volume of the ${ }^{18}$ F-FDG activity in the lesion and TLG represents the sum of the SUV within the lesion. Furthermore, glucose metabolic activity is positively correlated to tumor cellularity $[19,20]$.

Therefore, our study aimed to investigate the correlation between FDG parameters and ADC values, and focused, in-depth, on finding out if there is a correlation between tumor metabolic activity and cellularity, represented by ADC and SUVmax, TLG, and MTV, as well as assessing the ability of these imaging parameters to determine tumor aggressiveness, by predicting lymph node involvement.

\section{Materials and Methods}

\subsection{Patients and Demographics}

The Clinical Center, Regional and Local Research Ethics Committee (CCRLREC), University of Pecs Doctoral School of Health Sciences, and Somogy Megyei Kaposi Mor Educational Hospital, Pecs, Hungary (Approval Number: IG/04866.000/2020) approved this retrospective study [11]. The informed consent requirement was waived and confirmed by the (CCRLREC), and all methods were carried out following the applicable guidelines and laws (Declaration of Helsinki). From May 2016 to June 2019, 109 patients with confirmed HNC had their disease staged and assessed by 18F-FDG PET/MRI. (1) Patients had to have untreated main HNC, (2) they had to have PET/CT and PET/MRI with DWI sequences, 
and (3) they had to be non-smokers, as well as a single tracer injection session. Exclusion criteria: (1) patients who had non-measurable ADC, or FDG parameters; (2) patients with motion artefact or bad image quality. Finally, a total of 71 patients were included in our study, see Table 1 . Biopsy was the gold standard method for malignancy confirmation for all patients after PET/MRI examination.

Table 1. Patient demographics.

\begin{tabular}{cc}
\hline Number of Patients & $\mathbf{7 1}$ \\
\hline Mean Age (y) & $(61.6 \pm 0.8)$ \\
Men & $49(69.0 \%)$ \\
Women & $22(31.0 \%)$ \\
Histologic Grade & \\
Grade 1 & $12(16.9 \%)$ \\
Grade 2 & $41(57.7 \%)$ \\
Grade 3 & $18(20.4 \%)$ \\
Localization & \\
Pharyngeal & $32(45.1 \%)$ \\
Laryngeal & $15(21.1 \%)$ \\
Oral & $22(33.8 \%)$ \\
T category & \\
T1 & $4(5.6 \%)$ \\
T2 & $19(26.8 \%)$ \\
T3 & $26(36.6 \%)$ \\
T4 & $22(31.0 \%)$ \\
N category & \\
N0 & $10(14.1 \%)$ \\
N1 & $9(12.7 \%)$ \\
N2 & $45(63.4 \%)$ \\
N3 & $7(9.9 \%)$ \\
M Category & \\
M0 & $63(88.7 \%)$ \\
M1 & $8(11.3 \%)$ \\
N groups & $61(85.9 \%)$ \\
N+ & $10(14.1 \%)$ \\
N- & \\
\hline & \\
\hline
\end{tabular}

\subsection{PET/MRI Imaging}

The work strategy and procedure have been published elsewhere [11,12,21]. In brief, the examinations were conducted in a dedicated PET/MRI (3 T) equipment (Biograph mMR, Siemens AG, Erlangen, Germany). Patients were instructed to fast for at least $6 \mathrm{~h}$ and had their blood sugar levels checked to guarantee euglycemia before receiving the 18F-FDG injection intravenously. PET/MRI was conducted in the supine posture, images were captured with head and neck coils. PET/MRI parameters were also included (ADC, SUV, TLG, and MTV). MRI sequences were T2-weighted TSE turbo inversion recovery magnitude (TIRM) (TR/TE/TI 3300/37/220 ms, FOV: $240 \mathrm{~mm}$, slice thickness: $3 \mathrm{~mm}$, $224 \times 320)$ coronal plan, T1-weighted turbo spin-echo (TSE) (TR/TE 800/12 ms, FOV: $200 \mathrm{~mm}$, slice thickness: $4 \mathrm{~mm}, 224 \times 320)$, and T1-weighted TSE Dixon fat suppression (FS) (TR/TE 6500/85 ms, FOV: 200 mm, slice thickness: 4 mm, $256 \times 320)$ transversal and were acquired without an intravenous contrast agent [11,12,21].

\subsection{Image Analysis}

All methods of image analysis were previously published [11,12,21]. In short, a fixed 2.5 threshold of SUV was used for tumor SUVmax for both MTV and TLG, as proposed by Pak et al. [13]. DWI measurements were previously mentioned [12,21]. 'Avg' represents the average ADC values for all voxels within the ROI and 'Dev' Represents the standard deviation, see Figure 1. 

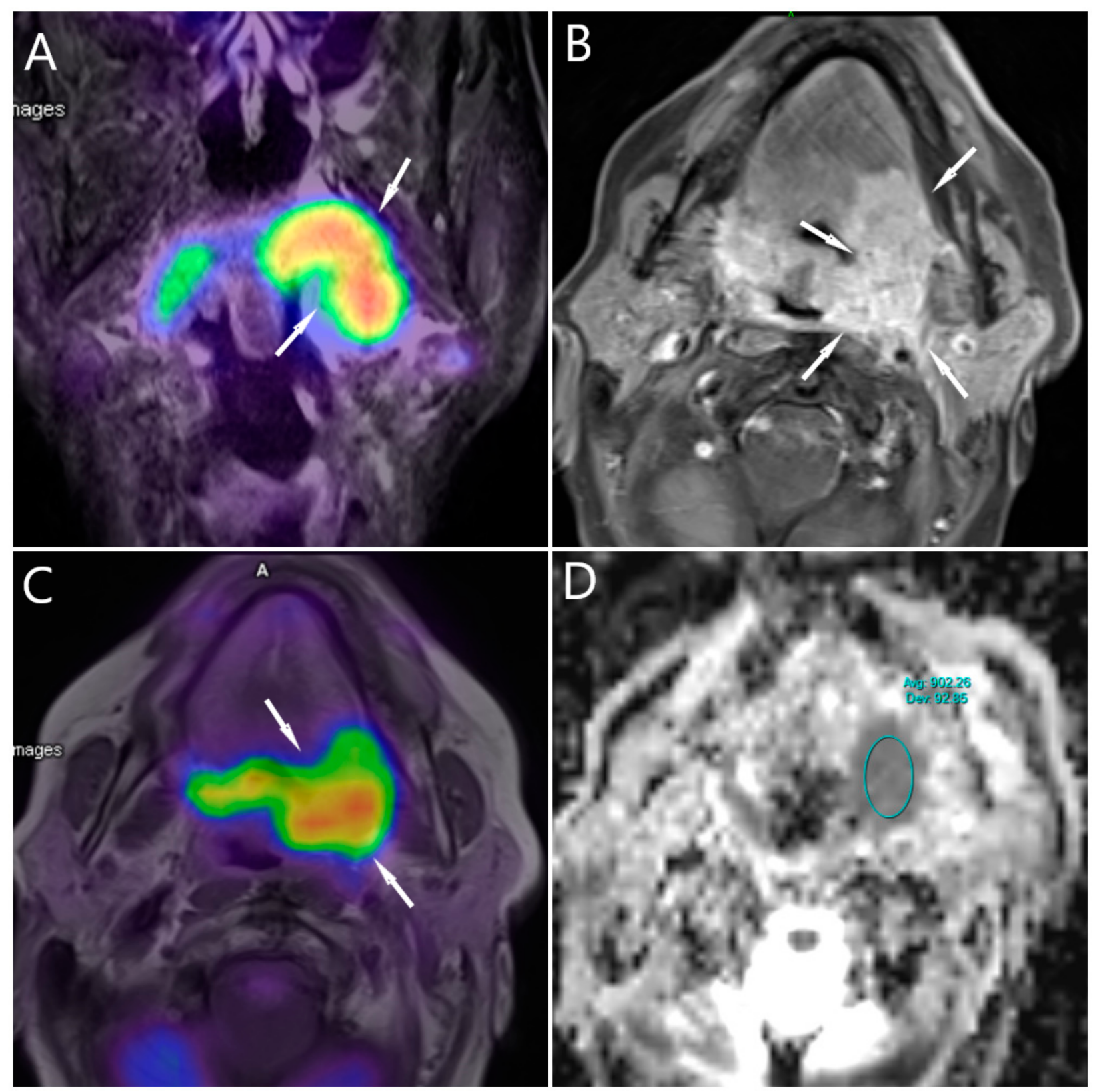

Figure 1. (A-D) and ${ }^{18}$ F-FDG measurements of 67 male patients with Oropharyngeal carcinoma. (A) T2-PET_tirm coronal MRI shows the intensive FDG accumulation (arrow). (B) T1-TSE-sagittal shows the extent of the tumor, lateral pharyngeal wall into the tongue root to the left tongue body (arrows). (C) T1-PET fused image shows the ROI within the tumor (arrows), and (D) DWI/ADC map showing the average and standard deviation of the ADC value.

\subsection{Statistical Analysis}

SPSS 25 was used to conduct a statistical analysis (IBM SPSS Statistics, Armonk, NY, USA). For variables with a normal distribution, descriptive statistics (mean and standard deviation) were used; whereas, for variables with a non-normal distribution, median and interquartile ranges were used. The Spearman rank correlation (r) was used to estimate the association between ${ }^{18}$ F-FDG parameters and ADC values and tumor size (continuous variable). ANOVA or a Kruskal-Wallis test were performed on the clinicopathological features that may affect the 18F- FDG and ADC of the tumor. By combining variables with $p$ $<0.1$ in a univariate analysis, a multiple linear regression analysis was used to find those that were independently linked with imaging parameters. To transform statistically significant 
differences in the univariate analysis into the multivariate linear regression model, we used a transforming function to convert variables with non-normal distribution into a normal distribution, then the factors were added one by one (Stepwise). A Mann-Whitney test and independent-sample T-test were applied to the imaging parameters after the patients were grouped based on lymph node involvement into positive $(\mathrm{N}+)$ and negative lymph nodes $(\mathrm{N}-)$. A $p$-value $<0.05$ indicated a statistically significant result.

\section{Results}

A summary of the measurements can be found in Supplementary file (Table S1). Spearman's correlation coefficient was applied on ${ }^{18} \mathrm{~F}-\mathrm{FDG}$ parameters and ADC values; the results show that ${ }^{18}$ F-FDG parameters (SUVmax, TLG and, MTV) were not correlated with ADC values $(\mathrm{r}=-0.184, p=0.125, \mathrm{r}=-0.182, p=0.248$, and $\mathrm{r}=-0.037, p=0.756)$, respectively. A summary of correlations is shown in Table 2 and Figure $2 \mathrm{~A}-\mathrm{C}$.

Table 2. Summary of correlations between FDG and DWI imaging parameters.

\begin{tabular}{rcccccc}
\hline & Parameter & ADC & SUVmax & TLG & MTV & Tumor Size \\
\hline \multirow{2}{*}{ ADC } & Spearman (rho) & & -0.184 & -0.182 & -0.037 & -0.088 \\
& Sig. (2-tailed) & & -0.125 & 0.129 & 0.756 & 0.464 \\
SUVmax & Spearman (rho) & & & & & $\mathbf{0 . 4 5 6}$ \\
& Sig. (2-tailed) & & & & $\mathbf{0 . 0 0 0}$ \\
TLG & Spearman (rho) & Sig. (2-tailed) & & & & $\mathbf{0 . 7 9 4}$ \\
& Spearman (rho) & & & & $\mathbf{0 . 0 0 0}$ \\
MTV & Sig. (2-tailed) & & & $\mathbf{0 . 7 3 9}$ \\
& & & & $\mathbf{0 . 0 0 0}$ \\
\hline
\end{tabular}

* Significant at a level of 0.05 , significant result in bold.

Moreover, the Spearman correlation coefficient was used to assess the correlation between 18F-FDG and tumor size (tumor size was measured as the maximum diameter of the tumor in pathologic results, mean size was $49.8 \pm 2.5 \mathrm{~mm}$ ). The results show that 18F-FDG parameters (SUVmax, TLG and MTV) were significantly and positively correlated with tumor size $(\mathrm{r}=0.456, p=0.001 ; \mathrm{r}=0.794, p=0.001$; and $\mathrm{r}=0.739, p=0.001)$, respectively. $\mathrm{ADC}$, on the other hand, showed no significant correlation with tumor size $(\mathrm{r}=-0.088, p=$ 0.464 ), see Table 2.

For a clinicopathological comparison, we compared primary tumor FDG (SUVmax, MTV, and TLG) and ADC parameters with sex, $T$ stages, N stages, M stages (7th Edition American Joint Committee on Cancer pathological staging criteria), [14] localization, and the degree of differentiation (grades). The results show that $\mathrm{N}$ stages were correlated with higher SUVmax, $(p=0.023)$. T stages and N stages were correlated with TLG values $(p=0.006$ and $p=0.033$, respectively). T stages were correlated with MTV values $(p=$ 0.001). Lower ADC, on the other hand, was found to be correlated with the degree of differentiation $(p=0.05)$, with a tendency for ADC to correlate with $\mathrm{N}$ stages, $(p=0.092)$. No other significant correlations were observed, $(p>0.05)$ for all parameters, see Table 3. 

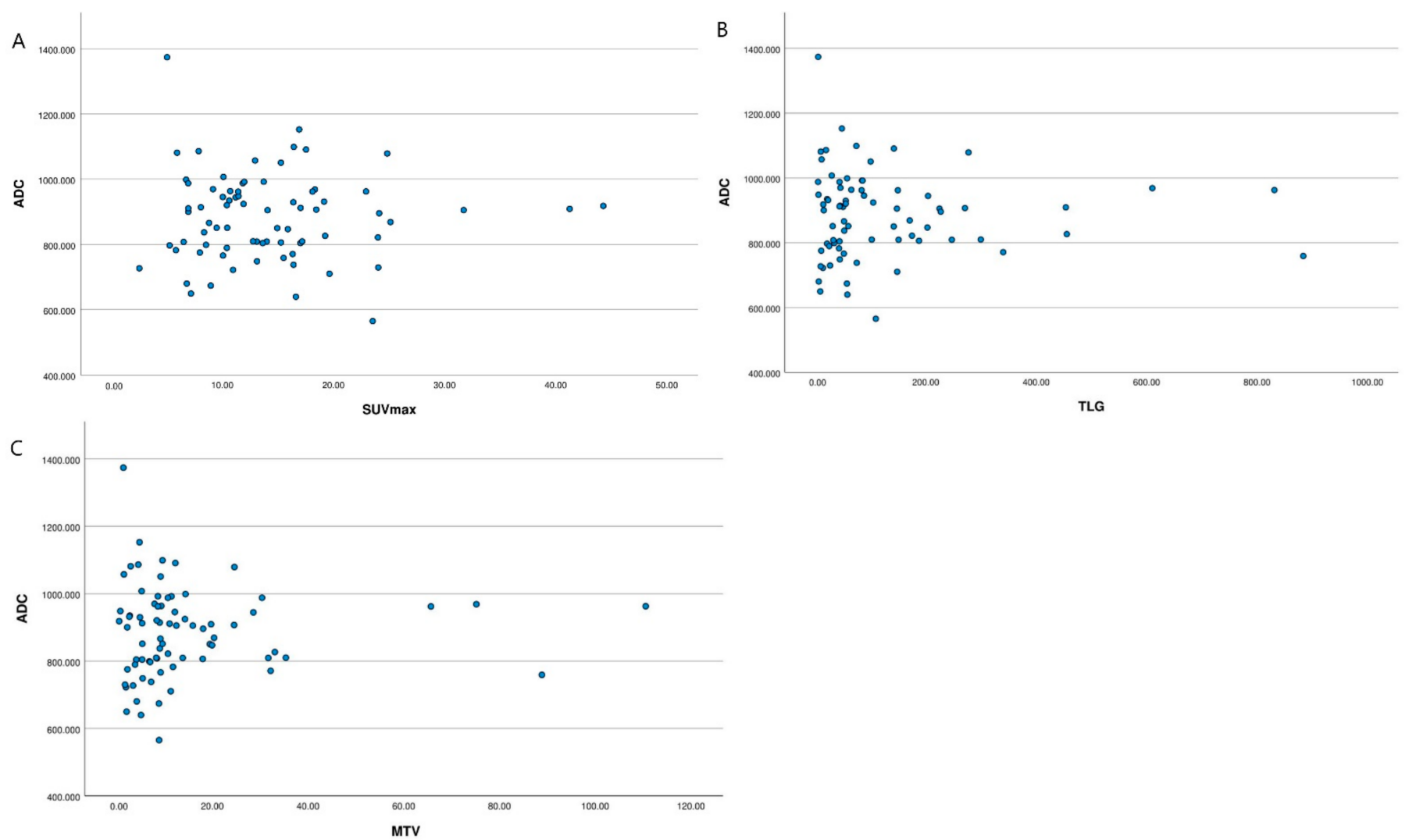

Figure 2. Scatter diagram showing the correlation between the ADCmean and (A) SUVmax, (B) TLG and (C) MTV. No significant linear correlation was observed between ADCmean and any of the 18F-FDG parameters, $p>0.05$.

Table 3. Clinicopathological comparison with FDG and DWI imaging parameters.

\begin{tabular}{clccc}
\hline Grouping & SUVmax & TLG & MTV & ADC \\
\hline SEX & $p=0.314$ & $p=0.522$ & $p=0.784$ & $p=0.897$ \\
T stages & $p=0.267$ & $p=\mathbf{0 . 0 0 6}$ & $p=\mathbf{0 . 0 0 1}$ & $p=0.880$ \\
N stages & $p=0.023$ & $p=0.033$ & $p=0.605$ & $p=0.092$ \\
M stages & $p=0.283$ & $p=0.785$ & $p=0.913$ & $p=0.347$ \\
Grades & $p=0.233$ & $p=0.310$ & $p=0.713$ & $p=0.050$ \\
Localization & $p=0.389$ & $p=0.128$ & $p=0.367$ & $p=0.270$ \\
\hline
\end{tabular}

Kruskal-Wallis for multi-categorical variables (T stages, $\mathrm{N}$ stages, localization, and tumor grades) and MannWhitney test for two categorical variables (sex, M stages) were used with (SUVmax, TLG and MTV). ANOVA and independent sample $t$-test were used with ADC values. Significant results are highlighted in bold. 
Multiple regression was recruited for factors that showed a statistically significant correlation in univariate analysis, to investigate the factor that influences the change in (SUVmax, TLG, MTV, and ADC). The results showed that tumor size and N stage were independent factors influencing SUVmax, $(p=0.001$ and $p=0.008$, respectively). Tumor size was an independent factor influencing TLG and MTV ( $p=0.001$ and $p=0.001$, respectively). Tumor grade was found to be an independent influencing factor of ADC ( $p=0.05)$. Table 4 .

Table 4. Multiple Regression Analysis Showing the Effects of Prognostic Factors on 18f-FDG parameters.

\begin{tabular}{cccc}
\hline Prognostic Factors & B & T & $p$ Value \\
\hline SUVmax & & & \\
Tumor size & 0.409 & 3.333 & $\mathbf{0 . 0 0 1} *$ \\
T stages & N/A & N/A $/ A$ \\
N stages & 0.227 & 1.995 & $\mathbf{0 . 0 2 2} *$ \\
TLG & & & \\
Tumor size & 0.767 & 8.988 & $\mathbf{0 . 0 0 0} *$ \\
T stages & -0.050 & -0.598 & 0.552 \\
N stages & 0.119 & 1.500 & 0.138 \\
MTV & & & $\mathbf{0 . 0 0 0}$ \\
Tumor size & 0.662 & 6.857 & 0.146 \\
T stages & 0.140 & 1.473 & $\mathrm{~N} / \mathrm{A}$ \\
N stages & $\mathrm{N} / \mathrm{A}$ & $\mathrm{N} / \mathrm{A}$ & 0.069 \\
ADC & & & $\mathbf{0 . 0 4 5}$ \\
N stages & 0.043 & 2.042 & \\
Tumor grades & -0.021 & -1.846 &
\end{tabular}

* Significant result; N/A: Not assessed. Significant results are highlighted in bold.

When removing the effect of the tumor size, SUVmax was correlated with $\mathrm{N}$ stages $(p=0.011)$, but not with T stages $(p=0.838)$; TLG was significantly correlated with both T stage and $\mathrm{N}$ stages ( $p=0.018$ and $p=0.034)$; and MTV was correlated to T stages $(p=0.001)$.

To investigate the ability of FDG and ADC parameters to predict lymph node involvement, we classified the patients based on lymph node involvement into negative and positive groups $(\mathrm{N}-$ and $\mathrm{N}+$ ) and compared them using these parameters. Our results show that SUVmax revealed statistically significant differences $(p=0.004)$; while TLG, MTV and ADC did not $(p>0.05)$. Figure 3A-D. 

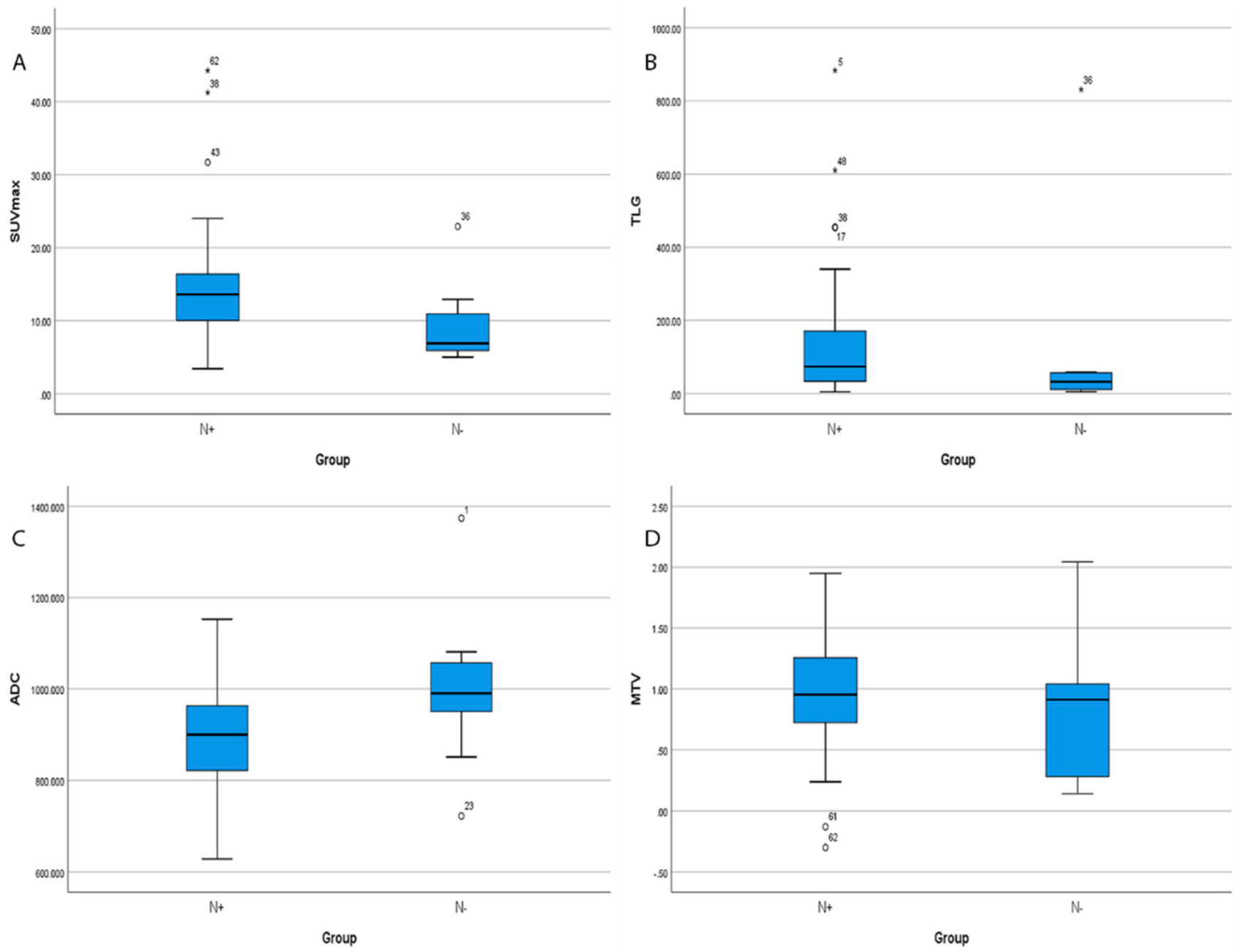

Figure 3. Boxplots displaying the distribution of SUVmax, TLG, ADC and MTV (A-D) according to lymph nodes status. (A) SUVmax values of positive lymph nodes tumors were significantly higher than those lymph nodes negative tumors $(p=0.004)$. (B) TLG show no significant difference between positive and negative lymph node $(p=0.084)$. (C) ADC values of positive lymph nodes tumors were not significantly between positive and negative lymph nodes $(p=0.074)$ and finally, (D) MTV positive lymph nodes tumors and negative lymph nodes tumors were not statistically significant difference $(p=0.342)$.

\section{Discussion}

The present study demonstrated that PET/MR provides valuable imaging data for HNC patients. Various pathological factors were associated with PET/MR results and may have a role in the evaluation of the prognosis of patients with HNC. PET/MRI offers different imaging data for studying tumor microstructure environments. Previous data demonstrated an inverse correlation between ADC value, derived from DWI, and cellularity [3-5,8]. FDG imaging parameters, on the other hand, were found to be positively correlated with cellularity $[15,19,20]$. Although the glucose metabolism and cellularity of tissue are two different biological biomarkers of a tumor, an inverse correlation between 18F-FDG and DWI has been suggested [15]. This hypothesis was proposed because both 18F-FDG and ADC were correlated with tumor cellularity [16].

In our study, the results showed that FDG uptake parameters (SUVmax, TLG, and MTV) were not significantly correlated with the ADC values. Similarly to Min et al., who found no significant correlation between ADCmean with SUVmax and SUVmean, no significant correlation was found between ADCmean and both MTV and TLG [22]. Surov 
et al., in a recent study, reported no significant correlation between ADCmean and SUVmax or SUVmean [23], and others [24-27].

On the other hand, contrarily to our results, Nunez et al. observed, in their study of HNSCC, a significant inverse correlation between the SUV and the ADC [27]. Nakajo et al. also observed that SUVmax was correlated inversely with ADCmean [28]. Han et al. reported a slightly significant inverse correlation between SUV and ADC. They also found a negative significant correlation between ADC and TLG [29].

Our explanation for the lack of correlation is the fact that both imaging parameters explain different tissue microstructures characteristics, where DWI assesses the water molecule motion in the tissue and is affected by the cellularity, proliferation rate, and cell counts, which in clinical use are affected by ROI size placement and interobserver variability [30]. On the other hand, metabolic activity was found to be independent of tumor size and shape, because the tumor is segmented by adaptive thresholding [16].

Furthermore, the tumor's clinicopathological characteristics were correlated to the imaging parameters, and the results reveal different correlations, as such; the primary tumor SUVmax was significantly correlated with the N stages; higher values of SUVmax were found in patients with a higher $\mathrm{N}$ stage. According to Zheng et al., there was a positive significant correlation between lymph nodes status and SUVmax; a higher SUVmax resulted in more lymph node metastasis, which means that SUVmax has a predictive role in lymph node diagnosis [31]. Micco et al. reported a significant correlation between lymph node occurrence and SUVmax and TLG [32]. Morand et al. observed similar results, where higher lymph node involvement was found in patients with higher primary tumor SUVmax [33]. In the same study, the authors reported that TLG did not correlate with lymph node status [33]. In our study, no significant correlation was observed between MTV and lymph node status, a similar result was reported by Morand et al. [33] and Chan et al. [34]. In summary, $\mathrm{N}$ stages and tumor size were independent factors influencing SUVmax. Tumor size and tumor T stages were independent factors influencing TLG and MTV. Thus, SUVmax might be a promising imaging biomarker to predict tumor aggressiveness.

ADC, on the other hand, shows a significant correlation with the tumor degree of differentiation, this results from the fact that a higher-grade tumor (G3) shows more restriction to water molecules, which as a result decreases the ADC value. Additionally, ADC did not show any significant correlation with $\mathrm{T}$ stages, $\mathrm{N}$ stages, or tumor size. Similar results were found by Nakajo et al. [28]. Moreover, other authors revealed the same findings $[35,36]$. In contrast, Abdel Razek et al., in their study of Nasopharyngeal carcinoma have reported a statistically significant difference between primary tumor ADC and nodal involvement [4]. While in our study, the explanation of different results was due to the heterogeneity of the patient's sample, which contained multiple primary tumor localization, and, thus, different anatomical and histological components were involved.

Although several studies have investigated the diagnostical role of 18F-FDG and ADC for determining tumor aggressiveness in different cancers [32,37-42], none of the studies have compared the efficacy of different PET/MRI imaging biomarkers in HNC tumor aggressiveness prediction. Thus, to our knowledge, this is the first study to compare PET/MRI system-derived imaging parameters in lymph node involvement in HNC. Our results show that SUVmax was found to be able to differentiate between the two lymph node groups $(\mathrm{N}+$ and $\mathrm{N}-$ ) based on the primary tumor measurements, which as a result might help to predict tumor development and prognosis. The importance of the successful prediction of tumor aggressiveness and lymph node involvement might help in daily practice, to increase the effectiveness of the therapy.

Based on our study results and findings, there were several correlations between PET/MRI imaging parameters and clinical tumor characteristics, and we suggest that glucose metabolism assessed by 18F-FDG and cellularity assessed by ADC have different roles in cancer evaluation; therefore, we recommend PET/MRI as a combined examination, rather than PET or MRI alone. 
As for this study's limitations, first is the heterogeneity of the tumor localization. Second, our study focused on the search for a correlation between 18F-FDG, ADC, and histopathological features only in HNC. Third, associations with other functional tumor parameters such as apoptosis factors were not analyzed. Fourth, the design of the study was retrospective.

\section{Conclusions}

Our results revealed no linear correlation between FDG PET and ADC MR parameters. FDG PET-based glucose metabolic and DWI MR derived cellularity data may represent different biological aspects of HNC tumors, and simultaneous PET/MR imaging could provide complementary diagnostic information. SUVmax showed a higher accuracy in predicting tumor aggressiveness than DWI.

Supplementary Materials: The following supporting information can be downloaded at: https: / / www.mdpi.com/article/10.3390/cancers14030847/s1, Table S1: Summary of measurements.

Author Contributions: O.F.: designed the study, O.F. and T.P.: collected and processed the data. D.S. and Á.K.: segmented FDG. measurements and generated the figures. O.F.: conducted data collection and processing, statistical analysis, and wrote the paper. T.Z.: review the draft, A.K. C.Z., and I.R.: discussed the results and contributed to the final form of the article and approved the last version for publication. All authors have read and agreed to the published version of the manuscript.

Funding: This research received no external funding.

Institutional Review Board Statement: Regional and Local Research Ethics Committee (CCRLREC), Doctoral School of Health Sciences, University of Pecs, and Somogy Megyei Kaposi Mor Educational Hospital, Pecs, Hungary (Approval Number: IG/04866.000/2020).

Informed Consent Statement: The requirement for informed consent was waived and confirmed by the (CCRLREC), due to the retrospective nature, and all methods were carried out following the relevant guidelines and regulations (Declaration of Helsinki).

Data Availability Statement: The datasets used and/or analyzed during the current study are available from the corresponding author on reasonable request.

Acknowledgments: We thank our colleagues from the Dr. József Baka Diagnostic, Radiation Oncology, Research and Teaching Center, Medicopus Non-Profit Ltd., "Moritz Kaposi” Teaching Hospital, Kaposvár, Hungary and Oncoradiology, Csolnoky Ferenc County Hospital, Veszprém, Hungary, who provided insight and expertise that greatly assisted the research, although they may not agree with all of the interpretations of this paper.

Conflicts of Interest: All authors declare no competing interests.

$\begin{array}{ll}\text { Abbreviations } \\ \text { PET/MRI } & \begin{array}{l}\text { Positron Emission Tomography/Magnetic Resonance Imaging } \\ \text { SUV }\end{array} \\ \text { Standardized Uptake Value } \\ \text { MTV } & \text { Metabolic Tumor Volume } \\ \text { TLG } & \text { Metabolic Tumor Volume } \\ \text { CRT } & \text { Chemo-Radiotherapy } \\ \text { HCC } & \text { Head and Neck Cancer } \\ \text { FDG } & \text { Fluorodeoxyglucose } \\ \text { AJCC } & \text { American Joint Committee on Cancer } \\ \text { (G) } & \text { Grade }\end{array}$

\section{References}

1. Pace, L.S.M.; Nicolai, E.; Aiello, M.; Catalano, O.A. Whole-body PET/MRI in oncology: Current status and clinical applica-tions. Transl. Imaging 2013, 1, 31-44. [CrossRef]

2. Queiroz, M.A.; Hüllner, M.; Kuhn, F.; Huber, G.; Meerwein, C.; Kollias, S.; Von Schulthess, G.; Veit-Haibach, P. Use of diffusionweighted imaging (DWI) in PET/MRI for head and neck cancer evaluation. Eur. J. Pediatr. 2014, 41, 2212-2221. [CrossRef] 
3. Becker, M.; Zaidi, H. Imaging in head and neck squamous cell carcinoma: The potential role of PET/MRI. Br. J. Radiol. 2014, 87, 20130677. [CrossRef] [PubMed]

4. Razek, A.A.K.A.; Kamal, E. Nasopharyngeal carcinoma: Correlation of apparent diffusion coeffecient value with prognostic parameters. Radiol. Med. 2013, 118, 534-539. [CrossRef]

5. Surov, A.; Meyer, H.J.; Wienke, A. Correlation between apparent diffusion coefficient (ADC) and cellularity is different in several tumors: A meta-analysis. Oncotarget 2017, 8, 59492-59499. [CrossRef] [PubMed]

6. Herneth, A.M.; Guccione, S.; Bednarski, M. Apparent Diffusion Coefficient: A quantitative parameter for in vivo tumor characterization. Eur. J. Radiol. 2003, 45, 208-213. [CrossRef]

7. Jeh, S.K.; Kim, S.H.; Kim, H.S.; Kang, B.J.; Jeong, S.H.; Yim, H.W.; Song, B.J. Correlation of the apparent diffusion coefficient value and dynamic magnetic resonance imaging findings with prognostic factors in invasive ductal carcinoma. J. Magn. Reson. Imaging 2010, 33, 102-109. [CrossRef]

8. Hayashida, Y.; Hirai, T.; Morishita, S.; Kitajima, M.; Murakami, R.; Korogi, Y.; Makino, K.; Nakamura, H.; Ikushima, I.; Yamura, M.; et al. Diffusion-weighted Imaging of Metastatic Brain Tumours: Comparison with Histologic Type and Tumour Cellularity. Am. J. Neuroradiol. 2006, 27, 1419-1425. Available online: http://www.ajnr.org/content/27/7/1419.abstract (accessed on 22 October 2021).

9. Zhang, S.C.; Bao, Y.Y.; Zhou, S.H.; Shang, D.S. Application value of diffusion weighted magnetic resonance imag-ing in head and neck cancer. Int. J. Clin. Exp. Med. 2016, 9, 16747-16752.

10. Srinivasan, A.; Dvorak, R.; Perni, K.; Rohrer, S.; Mukherii, S. Differentiation of Benign and Malignant Pathology in the Head and Neck Using 3T Apparent Diffusion Coefficient Values: Early Experience. Am. J. Neuroradiol. 2008, 29, 40-44. [CrossRef]

11. Freihat, O.; Tóth, Z.; Pintér, T.; Kedves, A.; Sipos, D.; Cselik, Z.; Lippai, N.; Repa, I.; Kovács, Á. Pre-treatment PET/MRI based FDG and DWI imaging parameters for predicting HPV status and tumor response to chemoradiotherapy in primary oropharyngeal squamous cell carcinoma (OPSCC). Oral Oncol. 2021, 116, 105239. [CrossRef] [PubMed]

12. Kedves, A.; Tóth, Z.; Emri, M.; Fábián, K.; Sipos, D.; Freihat, O.; Tollár, J.; Cselik, Z.; Lakosi, F.; Bajzik, G.; et al. Predictive Value of Diffusion, Glucose Metabolism Parameters of PET/MR in Patients with Head and Neck Squamous Cell Carcinoma Treated with Chemoradiotherapy. Front. Oncol. 2020, 10, 1484. [CrossRef]

13. Pak, K.; Cheon, G.J.; Nam, H.; Kim, S.; Kang, K.W.; Chung, J.; Kim, E.E.; Lee, D.S. Prognostic value of metabolic tumour volume and total lesion glycolysis in head and neck cancer: A systematic review and meta-analysis. J. Nucl. Med. 2014, 55, 884-890. Available online: http:/ / www.academia.edu/download/46307953/Prognostic_Value_of_Metabolic_Tumour_Volu2016060717979-obn7np.pdf (accessed on 23 September 2018). [CrossRef]

14. Edge, S.B.; Compton, C.C. The American Joint Committee on Cancer: The 7th Edition of the AJCC Cancer Staging Manual and the Future of TNM. Ann. Surg. Oncol. 2010, 17, 1471-1474. [CrossRef]

15. Vandecaveye, V.; De Keyzer, F.; Poorten, V.V.; Dirix, P.; Verbeken, E.; Nuyts, S.; Hermans, R. Head and Neck Squamous Cell Carcinoma: Value of Diffusion-weighted MR Imaging for Nodal Staging. Radiology 2009, 251, 134-146. [CrossRef] [PubMed]

16. Deng, S.; Wu, Z.; Wu, Y.; Zhang, W.; Li, J.; Dai, N.; Zhang, B.; Yan, J. Meta-analysis of the correlation between apparent diffusion coefficient and standardized uptake value in ma-lignant disease. Contrast Media Mol. Imaging 2017, 2017, 4729547. Available online: http:/ / www.epistemonikos.org/documents/2ffc3010315c57bfa650e08188580270f3fc4105 (accessed on 16 January 2022). [CrossRef] [PubMed]

17. Kim, Y.-I.; Paeng, J.C.; Cheon, G.J.; Suh, K.-S.; Lee, D.S.; Chung, J.-K.; Kang, K.W. Prediction of Posttransplantation Recurrence of Hepatocellular Carcinoma Using Metabolic and Volumetric Indices of 18F-FDG PET/CT. J. Nucl. Med. 2016, 57, 1045-1051. [CrossRef]

18. Sridhar, P.; Mercier, G.; Tan, J.; Truong, M.T.; Daly, B.; Subramaniam, R.M. FDG PET metabolic tumour volume seg- mentation and pathologic volume of pri-mary human solid tumours. AJR Am. J. Roentgenol. 2014, 202, 1114-1119. [CrossRef]

19. Bos, R.; Van Der Hoeven, J.J.; Van Der Wall, E.; Van Der Groep, P.; Van Diest, P.J.; Comans, E.F.; Joshi, U.; Semenza, G.L.; Hoekstra, O.S.; Lammertsma, A.A.; et al. Biologic Correlates of 18Fluorodeoxyglucose Uptake in Human Breast Cancer Measured by Positron Emission Tomography. J. Clin. Oncol. 2002, 20, 379-387. [CrossRef] [PubMed]

20. Ito, K.; Kato, T.; Ohta, T.; Tadokoro, M.; Yamada, T.; Ikeda, M.; Nishino, M.; Ishigaki, T.; Ito, K.; Gambhir, S. Fluorine-18 fluoro-2-deoxyglucose positron emission tomography in recurrent rectal cancer: Relation to tumour size and cellularity. Eur. J. Pediatr. 1996, 23, 1372-1377. [CrossRef]

21. Freihat, O.; Pinter, T.; Kedves, A.; Sipos, D.; Cselik, Z.; Repa, I.; Kovács, Á. Diffusion-Weighted Imaging (DWI) derived from PET/MRI for lymph node assessment in patients with Head and Neck Squamous Cell Carcinoma (HNSCC). Cancer Imaging 2020, 20, 56. [CrossRef] [PubMed]

22. Jeong, J.H.; Cho, I.H.; Chun, K.A.; Kong, E.J.; Kwon, S.D.; Kim, J.H. Correlation Between Apparent Diffusion Coefficients and Standardized Uptake Values in Hybrid 18F-FDG PET/MR: Preliminary Results in Rectal Cancer. Nucl. Med. Mol. Imaging 2016, 50, 150-156. [CrossRef]

23. Min, M.; Lee, M.T.; Lin, P.; Holloway, L.; Wijesekera, D.; Gooneratne, D.; Rai, R.; Xuan, W.; Fowler, A.; Forstner, D.; et al. Assessment of serial multi-parametric functional MRI (diffusion-weighted imaging and R2*) with18F-FDG-PET in patients with head and neck cancer treated with radiation therapy. Br. J. Radiol. 2016, 89, 20150530. [CrossRef] 
24. Fruehwald-Pallamar, J.; Czerny, C.; Mayerhoefer, M.E.; Halpern, B.S.; Eder-Czembirek, C.; Brunner, M.; Schuetz, M.; Weber, M.; Fruehwald, L.; Herneth, A.M. Functional imaging in head and neck squamous cell carcinoma: Correlation of PET/CT and diffusion-weighted imaging at 3 Tesla. Eur. J. Pediatr. 2011, 38, 1009-1019. [CrossRef]

25. Rasmussen, J.H.; Nørgaard, M.; Hansen, A.E.; Vogelius, I.R.; Aznar, M.C.; Johannesen, H.H.; Costa, J.; Engberg, A.M.; Kjaer, A.; Specht, L.; et al. Feasibility of Multiparametric Imaging with PET/MR in Head and Neck Squamous Cell Carcinoma. J. Nucl. Med. 2017, 58, 69-74. [CrossRef] [PubMed]

26. Varoquaux, A.; Rager, O.; Lovblad, K.-O.; Masterson, K.; Dulguerov, P.; Ratib, O.; Becker, C.D.; Becker, M. Functional imaging of head and neck squamous cell carcinoma with diffusion-weighted MRI and FDG PET/CT: Quantitative analysis of ADC and SUV. Eur. J. Pediatr. 2013, 40, 842-852. [CrossRef]

27. Covello, M.; Cavaliere, C.; Aiello, M.; Cianelli, M.; Mesolella, M.; Iorio, B.; Rossi, A.; Nicolai, E. Simultaneous PET/MR head-neck cancer imaging: Preliminary clinical experience and multiparametric evaluation. Eur. J. Radiol. 2015, 84, 1269-1276. [CrossRef]

28. Núñez, D.A.; Medina, A.L.; Iglesias, M.M.; Gomez, F.S.; Dave, A.; Hatzoglou, V.; Paudyal, R.; Calzado, A.; Deasy, J.O.; ShuklaDave, A.; et al. Multimodality functional imaging using DW-MRI and 18F-FDG-PET/CT during radiation therapy for human papillomavirus negative head and neck squamous cell carcinoma: Meixoeiro Hospital of Vigo Experience. World J. Radiol. 2017, 9, 17-26. [CrossRef]

29. Nakajo, M.; Nakajo, M.; Kajiya, Y.; Tani, A.; Kamiyama, T.; Yonekura, R.; Fukukura, Y.; Matsuzaki, T.; Nishimoto, K.; Nomoto, M.; et al. FDG PET/CT and diffusion-weighted imaging of head and neck squamous cell carcinoma: Comparison of prognostic significance between primary tumour standardized uptake value and apparent diffusion coefficient. Clin. Nucl. Med. 2012, 37, 475-480. [CrossRef] [PubMed]

30. Han, M.; Kim, S.Y.; Lee, S.J.; Choi, J.W. The Correlations Between MRI Perfusion, Diffusion Parameters, and 18F-FDG PET Metabolic Parameters in Primary Head-and-Neck Cancer. Medicine 2015, 94, e2141. [CrossRef]

31. Lambregts, D.M.J.; Beets, G.L.; Maas, M.; Curvo-Semedo, L.; Kessels, A.G.H.; Thywissen, T.; Beets-Tan, R.G.H. Tumour ADC measurements in rectal cancer: Effect of ROI methods on ADC values and interobserver variability. Eur. Radiol. 2011, 21, 2567-2574. [CrossRef] [PubMed]

32. Miccò, M.; Vargas, H.A.; Burger, I.A.; Kollmeier, M.A.; Goldman, D.A.; Park, K.; Abu-Rustum, N.R.; Hricak, H.; Sala, E. Combined pre-treatment MRI and 18F-FDG PET/CT parameters as prognostic biomarkers in patients with cervical cancer. Eur. J. Radiol. 2014, 83, 1169-1176. [CrossRef] [PubMed]

33. Morand, G.B.; Vital, D.G.; Kudura, K.; Werner, J.; Stoeckli, S.J.; Huber, G.F.; Huellner, M.W. Maximum Standardized Uptake Value (SUVmax) of Primary Tumor Predicts Occult Neck Metastasis in Oral Cancer. Sci. Rep. 2018, 8, 11817. [CrossRef] [PubMed]

34. Chan, W.K.; Mak, H.K.; Huang, B.; Yeung, D.W.; Kwong, D.L.-W.; Khong, P.-L. Nasopharyngeal carcinoma: Relationship between 18F-FDG PET-CT maximum standardized uptake value, metabolic tumour volume and total lesion glycolysis and TNM classification. Nucl. Med. Commun. 2010, 31, 206-210. [CrossRef]

35. Karan, B.; Pourbagher, A.; Torun, N. Diffusion-weighted imaging and18F-fluorodeoxyglucose positron emission tomography/computed tomography in breast cancer: Correlation of the apparent diffusion coefficient and maximum standardized uptake values with prognostic factors. J. Magn. Reson. Imaging 2015, 43, 1434-1444. [CrossRef]

36. Choi, B.B.; Kim, S.H.; Kang, B.J.; Lee, J.H.; Song, B.J.; Jeong, S.H.; Yim, H.W. Diffusion-weighted imaging and FDG PET/CT: Predicting the prognoses with apparent diffusion coefficient values and maximum standardized uptake values in patients with invasive ductal carcinoma. World J. Surg. Oncol. 2012, 10, 126. [CrossRef]

37. Yang, Z.; Wu, J.R.; Wei, L.L.; Liao, G.X.; Yang, C.J.; Jin, G.Q.; Xiao, G.Y.; Su, D.K. High standardized uptake values of 18F-FDG PET/CT imaging but not MRI correlates to pathology find-ings in patients with cervical cancer. Hell. J. Nucl. Med. 2019, 22, 96-102. [CrossRef]

38. Gong, J.; Wang, N.; Bian, L.; Wang, M.; Ye, M.; Wen, N.; Fu, M.; Fan, W.; Meng, Y. Cervical cancer evaluated with integrated 18F-FDG PET/MR. Oncol. Lett. 2019, 18, 1815-1823. [CrossRef]

39. Yu, Y.Y.; Zhang, R.; Dong, R.T.; Hu, Q.Y.; Yu, T.; Liu, F.; Luo, Y.H.; Dong, Y. Feasibility of an ADC-based radiomics model for predicting pelvic lymph node metastases in patients with stage IB-IIA cervical squamous cell carcinoma. Br. J. Radiol. 2019, 92, 20180986. [CrossRef]

40. Nerad, E.; Pizzi, A.D.; Lambregts, D.M.J.; Maas, M.; Wadhwani, S.; Bakers, F.C.H.; Bosch, H.C.M.V.D.; Beets-Tan, R.G.H.; Lahaye, M.J. The Apparent Diffusion Coefficient (ADC) is a useful biomarker in predicting metastatic colon cancer using the ADC-value of the primary tumor. PLoS ONE 2019, 14, e0211830. [CrossRef]

41. Xu, C.; Li, H.; Seng, D.; Liu, F. Significance of SUV Max for Predicting Occult Lymph Node Metastasis and Prognosis in Early-Stage Tongue Squamous Cell Carcinoma. J. Oncol. 2020, 2020, 6241637. [CrossRef] [PubMed]

42. Kato, F.; Kudo, K.; Yamashita, H.; Baba, M.; Shimizu, A.; Oyama-Manabe, N.; Kinoshita, R.; Li, R.; Shirato, H. Predicting metastasis in clinically negative axillary lymph nodes with minimum apparent diffusion coefficient value in luminal A-like breast cancer. Breast Cancer 2019, 26, 628-636. [CrossRef] [PubMed] 